\title{
A Weak Equivalence and Topological Entropy
}

By

\section{Taijiro OHNo}

\section{§1. Introduction}

In this paper we will investigate the topological entropies of mutually weakly equivalent topological flows. Roughly speaking, any two flows which are weakly equivalent to each other have the same orbits. So the notion of weak equivalence of flows is, in a sense, a generalization of time changes of flows. In [5] Totoki investigated time changes of flows from a measure theoretical point of view. Especially he showed that for metrical (=measure theoretical) flows time changes preserve the properties that the metrical entropy is zero, positive, finite or infinite respectively. Here we will be rather concerned with topological flows and their topological entropies.

First we will consider flows without fixed point. In this case we obtain a result analogous to Totoki's one. Namely, the properties that the topological entropy is zero, positive, finite or infinite respectively are invariant under weak equivalence of flows without fixed point (Theorem 1 in $\$ 3$ ). But this is not the case if the flows have fixed points. Indeed, we will construct a pair of flows with the same orbits and a fixed point one of which has a positive entropy and the other has zero entropy (Theorem 2 in \$4).

In the proof of these two theorems we will appeal to a measure theoretical method. The point is that the topological entropy is the supremum of metrical entopies with respect to all invariant Borel probability measures (Lemma 2 in §).

The idea of the construction of the example in Section 4 to prove Theorem 2 is as follows. Take a flow with a fixed point such that each orbit visits a neighbourhood of the fixed point infinitely often and that the ratio of the sojourn time in the neighbourhood is uniformly positive. One can construct such a flow with a positive topological entropy. Then, lowering the speed of the flow

Communicated by K. ITO, May 17, 1978.

* Department of Mathematics, Kyoto University. 
near the fixed point so that the ratio of the sojourn time converges to 1 , one can obtain a flow which has no finite invariant measure except the point measure at the fixed point. Thus this flow has zero entropy. We remark that in this example the original flow has an invariant Borel probability measure with a positive metrical entropy and that the modified flow has only a trivial invariant Borel probability measure.

In Section 2 we define the notion of weak equivalence of flows, explain the relation of the weak equivalence to the time change and then establish a correspondence between invariant ergodic probability measures of two flows with the same orbits (Proposition 1) as a preparation for the proof of Theorem 1. Sections 3 and 4 are devoted to the proof of our main results mentioned above.

\section{§. Weak Equivalence and Time Change}

Let $X$ be a compact metric space. A (topological) flow $\Phi=\left\{\varphi_{t} ;-\infty<t<\infty\right\}$ on $X$ is a one-parameter group of homeomorphisms of $X$ which is continuous in $(t, x)$. Given $\Phi$ and $\Psi$ flows on $X$ and $Y$ respectively, we say that $\Phi$ and $\Psi$ are weakly equivalent if there is a homeomorphism $\pi$ of $X$ onto $Y$ such that $\Phi$ and $\hat{\Phi}=\left\{\hat{\varphi}_{t}=\pi^{-1} \circ \psi_{t} \circ \pi ;-\infty<t<\infty\right\}$ have the same orbits i.e. $\left\{\varphi_{t}(x) ;-\infty<t<\infty\right\}$ $=\left\{\pi^{-1} \circ \psi_{t} \circ \pi(x) ;-\infty<t<\infty\right\}$ for all $x \in X$.

For a while let us assume further that $\Phi$ and $\hat{\Phi}$ have the same orbits with the same directions. Let $X_{0}$ denote the set of all $\Phi$-fixed points. We can define $\theta(t, x),-\infty<t<\infty, x \in X \backslash X_{0}$, with the following properties (cf. [2]):

i) $\varphi_{t}(x)=\pi^{-1} \circ \psi_{\theta(t, x)} \circ \pi(x)=\hat{\varphi}_{\theta(t, x)}(x)$,

ii) $\theta(t+s, x)=\theta\left(t, \varphi_{s}(x)\right)+\theta(s, x)$

iii) $\theta(0, x)=0$ and $\theta(t, x)$ is strictly increasing in $t$,

iv) $\theta(t, x)$ is continuous in $(t, x)$.

Thus we see that $\theta$ is an additive functional of $\Phi$ and that $\hat{\Phi}$ is a time changed flow of $\Phi$ in the sense of [5]. Under the situation stated above, we will study a relation of invariant measures. Let $\varepsilon(\Phi)$ denote the family of all $\Phi$-invariant ergodic Borel probability measures on $X$. Then we have

Proposition 1. Assume that the flows $\Phi$ and $\hat{\Phi}$ have the same orbits with the the same directions and they have no fixed point. Let $\theta$ be the additive functional of $\Phi$ obtained above. Then, for each $m \in \varepsilon(\Phi)$, 


$$
E_{\hat{m}}(f)=\frac{1}{E_{m}(\theta(1, x))} E_{m}\left(\int_{0}^{\theta(1, x)} f\left(\hat{\varphi}_{t}(x)\right) d t\right)^{1)}
$$

defines an $\hat{m} \in \varepsilon(\hat{\Phi})$. Then map $\wedge: m \in \varepsilon(\Phi) \rightarrow m \in \varepsilon(\hat{\Phi})$ is bijective and $G(\Phi, m)$ $=G(\hat{\Phi}, \hat{m})$ where $G(\Phi, m)=\left\{x \in X ; \lim _{T \rightarrow \infty} \frac{1}{T} \int_{0}^{T} f\left(\varphi_{t}(x)\right) d t=E_{m}(f)\right.$ for all continuous $\left.f\right\}$.

In order to prove the proposition we prepare the following

Lemma 1. For any continuous function $f$, we have

$$
\lim _{n \rightarrow \infty} \frac{1}{n} \int_{0}^{\theta(n, x)} f\left(\hat{\varphi}_{s}(x)\right) d s=\operatorname{ltm}_{n \leqslant \infty} \frac{1}{n} \int_{0}^{n} \int_{0}^{\theta\left(1, \varphi_{t}(x)\right)} f\left(\hat{\varphi}_{s} \circ \varphi_{t}(x)\right) d s d t
$$

if one of the limits exists.

Proof. Since $\int_{0}^{\theta(1, x)} f\left(\hat{\varphi}_{s}(x)\right) d s$ is continuous, for any $\varepsilon>0$ there is a $\delta>0$ such that the distance $d(x, y)<\delta$ implies

$$
\left|\int_{0}^{\theta(1, x)} f\left(\hat{\varphi}_{s}(x)\right) d s-\int_{0}^{\theta(1, y)} f\left(\hat{\varphi}_{s}(y)\right) d s\right|<\varepsilon
$$

The continuity of $\varphi_{t}(x)$ and the compactness of $X$ imply the existence of $\frac{1}{p}$ such that $d\left(\varphi_{\ell}(x), x\right)<\delta$ if $|t|<\frac{1}{p}$ for any $x \in X$. We put

$$
\begin{aligned}
I_{n} & =\frac{1}{n} \int_{0}^{n} \int_{0}^{\theta\left(1, \varphi_{t}(x)\right)} f\left(\hat{\varphi}_{s} \circ \varphi_{t}(x)\right) d s d t \\
& =\frac{1}{n} \sum_{k=0}^{n p-1} \int_{k / p}^{(k+1) / p} \int_{0}^{\theta\left(1, \varphi_{t}(x)\right)} f\left(\hat{\varphi}_{s} \circ \rho_{t}(x)\right) d s d t
\end{aligned}
$$

which is approximated by

$$
\Pi_{n}=\frac{1}{n p} \sum_{k=0}^{n p-1} \int_{0}^{\theta\left(1, \varphi_{k / p}^{(x))}\right.} f\left(\hat{\varphi}_{s} \circ \varphi_{k / x}(x)\right) d s
$$

as $\left|I_{n}-\Pi_{n}\right|<\varepsilon$. Since

$$
\int_{0}^{\theta\left(1, \varphi_{r}(x)\right)} f\left(\hat{\varphi}_{s} \circ \varphi_{r}(x)\right) d s=\int_{\theta(r, x)}^{\theta(r+1, x)} f\left(\hat{\varphi}_{s}(x)\right) d s
$$

we have

$$
\frac{\Pi_{n}=\frac{1}{n} \int_{\theta(1, x)}^{\theta(n, x)} f\left(\hat{\varphi}_{s}(x)\right) d s+\frac{1}{n p}\left[\sum_{k=0}^{p-1} \int_{\theta(k / p, x)}^{\theta(1, k)} f\left(\hat{\varphi}_{s}(x)\right) d s+\right.}{\text { 1) } E_{m}(f)=\int_{X} f(x) d m(x)}
$$




$$
\left.\sum_{k=0}^{p-1} \int_{\theta(n, x)}^{\theta(n+(k / p), x)} f\left(\hat{\varphi}_{s}(x)\right) d s\right]
$$

Now it is easy to see

$$
\left|\frac{1}{n} \int_{0}^{\theta(n, x)} f\left(\hat{\varphi}_{s}(x)\right) d s-\Pi_{n}\right| \rightarrow 0 \text { as } n \rightarrow \infty .
$$

Proof of Proposition 1. Take any $x \in G(\Phi, m)$. The limit of the right hand side of (1) exists and equals to

$$
\begin{aligned}
& E_{m}\left(\int_{0}^{\theta(1, x)} f\left(\hat{\varphi}_{s}(x)\right) d s\right) \text { for this } x \text {. Thus we have } \\
& \lim _{n \rightarrow \infty} \frac{1}{n} \int_{0}^{\theta(n, x)} f\left(\hat{\varphi}_{s}(x)\right) d s=E_{m}\left(\int_{0}^{\theta(1, x)} f\left(\hat{\varphi}_{s}(x)\right) d s\right)
\end{aligned}
$$

and putting $f \equiv 1$

$$
\lim _{n \rightarrow \infty} \frac{1}{n} \theta(n, x)=E_{m}(\theta(1, x))
$$

Therefore we have

$$
\lim _{n \rightarrow \infty} \frac{1}{\theta(n, x)} \int_{0}^{\theta(n, x)} f\left(\hat{\varphi}_{s}(x)\right) d s=E_{\hat{m}}(f) .
$$

For any $T>0$ define $n_{T}$ by

$$
\begin{aligned}
& \theta\left(n_{T}, x\right) \leqq T<\theta\left(n_{T}+1, x\right), \text { then } \lim _{T \rightarrow \infty} n_{T}=\infty \text {. Hence } \\
& \qquad \begin{aligned}
\lim _{T \rightarrow \infty} \frac{1}{T} \int_{0}^{T} f\left(\hat{\varphi}_{s}(x)\right) d s & =\lim _{T \rightarrow \infty} \frac{1}{\theta\left(n_{T}, x\right)} \int_{0}^{\theta\left(n_{T}, x\right)} f\left(\hat{\varphi}_{s}(x)\right) d s \\
& =E_{\hat{m}}(f) .
\end{aligned}
\end{aligned}
$$

Thus $x \in G(\hat{\Phi}, \hat{m})$ and so $G(\Phi, m) \subset G(\hat{\Phi}, \hat{m})$.

It is easy to see that $\hat{m}$ is a $\hat{\Phi}$-invariant probability measure. Since

$$
\hat{m}(G(\hat{\Phi}, \hat{m})) \geqq \hat{m}(G(\Phi, m))=\frac{E_{m}\left(\theta(1, x) 1_{G(\Phi, m)}(x)\right)}{E_{m}(\theta(1, x))}=1,
$$

$\hat{m}$ is ergodic for $\hat{\Phi}$. By the symmetry, there is a $P \in \varepsilon(\Phi)$ such that $G(\hat{\Phi}, \hat{m}) \subset$ $G(\Phi, P)$. But this implies $P=m$ and so $G(\Phi, m)=G(\hat{\Phi}, \hat{m})$.

Corollary 1. Under the same assumption as Proposition 1 , let $\hat{\theta}$ be the additive functional of $\hat{\Phi}$ such that $\hat{\varphi}_{t}(x)=\varphi_{\hat{\theta}(t, x)}(x)$. Then 


$$
\frac{1}{E_{\hat{m}}(\theta(1, x))} E_{\hat{m}}\left(\int_{0}^{\hat{\theta}(1, x)} f\left(\varphi_{t}(x)\right) d t\right)=E_{m}(f)
$$

for any $m \in \varepsilon(\Phi)$ where $\hat{m}$ is defined in Proposition 1 .

Corollary 2. Any time changed flow of a strictly ergodic (i.e. minimal and uniquely ergodic) flow is also strictly ergodic.

\section{§3. Weakly Equivalent Flows without Fixed Points}

We will prove the following

Theorem 1. Let $\Phi=\left\{\varphi_{t}\right\}$ and $\Psi=\left\{\psi_{t}\right\}$ be topological flows on compact metric spaces $X$ and $Y$ respectively. If $\Phi$ and $\Psi$ are weakly equivalent and they have no fixed point then we have

$$
h(\Phi)=C_{\Phi \Psi} h(\psi)
$$

where $h(\Phi)$ and $h(\Psi)$ are topological entropy of $\Phi$ and $\Psi$ respectively, and $C_{\Phi \Psi}$ is a finite positive number.

Remark. The topological entropy $h\left(\varphi_{t}\right)$ of a single map $\varphi_{t}$ was introduced in [1], and the following equality for a flow $\Phi=\left\{\varphi_{t}\right\}$ was proved by Sh. Ito [3];

$$
h\left(\varphi_{1}\right)=\frac{1}{|t|} h\left(\varphi_{t}\right) .
$$

We define naturally the topological entropy of a flow $\Phi=\left\{\varphi_{t}\right\}$ by $h(\Phi)=h\left(\varphi_{1}\right)$.

In order to prove the theorem we will apply a measure theoretical method. For a single map $\varphi_{1}$ we also define $\varepsilon\left(\varphi_{1}\right)$ as the set of all $\varphi_{1}$-invariant ergodic Borel probability measures on $X$.

Lemma 2. We have

$$
h\left(\varphi_{1}\right)=\sup _{\mu \in \mathbb{\varepsilon}\left(\varphi_{1}\right)} h_{\mu}\left(\varphi_{1}\right)=\sup _{m \in \mathbb{\varepsilon}(\Phi)} h_{m}\left(\varphi_{1}\right)
$$

where $h_{\mu}\left(\varphi_{1}\right)$ denotes the metrical entropy of $\varphi_{1}$ with respect to the measure $\mu$.

Proof. The first equality is well known (cf.[6]). We will prove the second one. The ergodic decomposition theorem for entropy ([4]) implies $h_{m}\left(\varphi_{1}\right) \leqq$ $\sup _{\left.\mu \in \mathbb{\operatorname { e } ( \varphi}, \varphi_{1}\right)} h_{\mu}\left(\varphi_{1}\right)$ for any $m \in \varepsilon(\Phi)$. To prove the converse inequality, for each $\mu \in \varepsilon\left(\varphi_{1}\right)$ and $t \in R$, we define $\mu_{t}(A)=\mu\left(\varphi_{t}(A)\right)$ and $m(A)=\int_{0}^{1} \mu_{t}(A) d t$. Then $\mu_{t} \in \varepsilon\left(\varphi_{1}\right)$ and $m \in \varepsilon(\Phi)$. Since $\left(X, \mu, \varphi_{1}\right)$ and $\left(X, \mu_{t}, \varphi_{1}\right)$ are isomorphic we have 
$h_{\mu_{t}}\left(\varphi_{1}\right)=h_{\mu}\left(\varphi_{1}\right)$. The concavity of the function $-x \log x$ implies

$$
h_{m}\left(\varphi_{1}\right) \geqq \int_{0}^{1} h_{\mu_{t}}\left(\varphi_{1}\right) d t=h_{\mu}\left(\varphi_{1}\right) .
$$

Proof of Theorem 1. Denoting $\hat{\Phi}=\left\{\hat{\varphi}_{t}=\pi^{-1} \circ \psi_{t} \circ \pi ;-\infty<t<\infty\right\}$ we have $h(\hat{\Phi})=h(\Psi)$. Let $X^{+}=\{x \in X ; \Phi$ and $\hat{\Phi}$ have the same direction at $x\}$ and $X^{-}=$ $\{x \in X ; \Phi$ and $\hat{\Phi}$ have the oposite direction at $x\}$. Then under the assumption of the theorem, $X^{+}$and $X^{-}$are both closed invariant sets, and so we have

$$
h(\Phi)=\max \left\{h\left(\Phi \mid X^{+}\right), h\left(\Phi \mid X^{-}\right)\right\}
$$

where $\Phi \mid X^{ \pm}$are the restrictions of $\Phi$ to $X^{ \pm}$respectively (cf.[1]). On the other hand we have $h\left(\varphi_{t}\right)=h\left(\varphi_{-t}\right)$. Therefore we may and do assume that $\Phi$ and $\hat{\Phi}$ have the same orbits with the same directions. Then we obtain an additive functional $\theta(t, x)$ of $\Phi$ such that $\varphi_{t}(x)=\hat{\varphi}_{\theta(t, x)}(x)$ as stated in Section 2. It is proved in [5] that for any $m \in \varepsilon(\Phi)$

$$
h_{\hat{m}}\left(\hat{\varphi}_{1}\right)=\frac{1}{E_{n \imath}(\theta(1, x))} h_{m}\left(\varphi_{1}\right)
$$

where $\hat{m} \in \varepsilon(\hat{\Phi})$ is defined in Proposition 1. We have

$$
h(\Phi)=\sup _{m \in \varepsilon(\Phi)} h_{m}\left(\varphi_{1}\right)=\sup _{m \in \varepsilon(\Phi)} E_{m}(\theta(1, x)) h_{\hat{m}}\left(\hat{\varphi}_{1}\right),
$$

and so putting $c_{1}=\min _{x \in X} \theta(1, x)$ and $c_{2}=\max _{x \in X} \theta(1, x)$

$$
\begin{aligned}
c_{1} h(\hat{\Phi}) & =c_{1} \sup _{\hat{m_{\in}} \in \mathcal{\varepsilon}(\hat{\Phi})} h_{\hat{m}}\left(\hat{\varphi}_{1}\right)=c_{1} \sup _{m_{m \in \mathbb{E}(\Phi)}} h_{\hat{m}}\left(\hat{\varphi}_{1}\right) \leqq h(\Phi) \\
& \leqq c_{2} \sup _{m \in \mathcal{E}(\Phi)} h_{\hat{m}}\left(\hat{\varphi}_{1}\right)=c_{2} h(\hat{\Phi}) .
\end{aligned}
$$

So, we have the conclusion of Theorem 1 .

\section{§4. Weakly Equivalent Flows with Fixed Points}

We will construct an example of a pair of flows which proves the following

Theorem 2. There exists a pair of weakly equivalent flows one of which has a positive topological entropy and the other has zero entropy.

Construction. We will construct an example as a pair of flows under functions.

Step 1. We will define a sequence $x^{*} \in\{0,1\}^{Z}$ such that (i) for all $k \in Z$ and $n \geqq 1$ we have $x^{*}(k) x^{*}(k+1) \cdots x^{*}\left(k+4 \cdot 3^{n}\right)>I_{n}=11 \cdots 1$ i.e. there are $2 n-1$ 
consecutive 1's in $x^{*}(k) x^{*}(k+1) \cdots x^{*}\left(k+4 \cdot 3^{n}\right)$, and (ii) for all $n \geqq 1$ there exist $2^{p_{n}}$ different words (sequences) of length $2 \cdot 3^{n-1}$ in $x^{*}$ where $p_{n}=\left(3^{n-1}+1\right) / 2$.

First we define $x^{+} \in\{1, \alpha\}^{N}$ as follows, where $\alpha$ is a symbol which will be replaced by 0 or 1 later. We define $x_{n}$ inductively. Put $x_{1}=1 \alpha$ and then $x_{2}=$ $x_{1} \tilde{x}_{1} x_{1}$ where $\tilde{x}_{1}=11$. We define $\tilde{x}_{n}$ by changing one $\alpha$ in $x_{n}$ to 1 so that $\tilde{x}_{n}>I_{n}$, and $x_{n+1}=x_{n} \tilde{x}_{n} x_{n}$. Let $x^{+}=\lim _{n \rightarrow \infty} x_{n}$. Then there are infinitely many $x_{n}$ in $x^{+}$for all $n \geqq 1$. The number of $\alpha$ in $x_{n}$ is $p_{n}=\left(3^{n-1}+1\right) / 2$.

Changing $\alpha$ 's in $x_{n}$ to 0 or 1 we can obtain $2^{p_{n}}$ different words of length $2 \cdot 3^{n-1}$. We replace $2^{p_{n}} x_{n}$ 's to those words in turn from the top of $x^{+}, n=1,2, \cdots$, and put 0 or 1 arbitrarily into the remaining $\alpha$ 's. Thus we obtain $x^{+}$in $\{0,1\}^{N}$. We put $x^{*}(k)=x^{+}(k)$ for $k \geqq 1, x^{*}(0)=1$ and $x^{*}(k)=x^{+}(-k)$ for $k \leqq-1$. Then it is easy to see that $x^{*}$ satisfies (i) and (ii).

Step 2. Let $T$ be the shift transformation of $\{0,1\}^{Z}$ and $X=$ the closure of $\left\{T^{n} x^{*} ; n \in Z\right\}$ in $\{0,1\}^{z}$ which is endowed with the product of the discrete topology. Then $(X, T)$ is a subshift of $\left(\{0,1\}^{z}, T\right)$. For any $\mu \in \varepsilon(X, T)$ there exists $x \in X$ such that

$$
\mu\left(\hat{I}_{n}\right)=\lim _{m \rightarrow \infty} \frac{1}{m} \sum_{i=0}^{m-1} 1_{\hat{I}_{n}}\left(T^{i} x\right)
$$

for all $n \geqq 1$, where $\hat{I}_{n}=\{x \in X ; x(k)=1,-n+1 \leqq k \leqq n-1\}$ and $1_{\hat{I}_{n}}$ is the indicator function of $\hat{I}_{n}$. Since $x$ is an accumulating point of $\left\{T^{n} x^{*} ; n \in Z\right\}$, it satisfies (i) in Step 1 and so we have

$$
\mu\left(\hat{I}_{n}\right) \geqq \frac{1}{4 \cdot 3^{n}}, \quad \text { for all } n \geqq 1
$$

On the other hand we have an estimation of the topological entropy of $T$;

$$
h(T)=\lim _{n \rightarrow \infty} \frac{1}{n} \log N_{n} \geqq \lim _{n \rightarrow \infty} \frac{1}{2 \cdot 3^{n-1}} \log 2^{p_{n}}=\frac{1}{4} \log 2>0,
$$

where $N_{n}$ denotes the number of different cylinder sets on $[0, n-1]$.

Step 3. Let $1 \in X$ denote the fixed point of $T$ such that $1(k)=1$ for all $k \in Z$. Put $X_{*}=X \backslash\{1\}$, which is a locally compact space. Let $\gamma$ be a positive continuous function on $X_{*}$. We define the quotient space $X_{*}^{\gamma}$ of $\{(x, u) ; 0 \leqq u \leqq$ $\left.r(x), x \in X_{*}\right\}$ by the equivalent relation $(x, r(x)) \sim(T x, 0)$. We also define a flow $\left\{\varphi_{t}^{\gamma}\right\}$ by

$$
\varphi_{t}^{\gamma}(x, u)=(x, u+t), \text { for }-u \leqq t<\gamma(x)-u
$$


Then $X_{*}^{\gamma}$ is a locally compact metric space and $\varphi_{t}^{\gamma}$ is a topological flow on $X_{*}^{\gamma}$.

Let $X^{\gamma}=X_{*}^{\gamma} \cup\{\Delta\}$ be a one point compactification of $X_{*}^{\gamma}$. Then $X^{\gamma}$ is a compact metric space. Notice that $\left(x_{n}, u_{n}\right) \rightarrow \Delta$ in $X^{\gamma}$ if and only if $x_{n} \rightarrow 1$ in $X$. Extending $\left\{\varphi_{t}^{\gamma}\right\}$ as $\varphi_{t}^{\gamma}(\Delta)=\Delta$ for all $-\infty<t<\infty$, we obtain a topological flow $\Phi^{\gamma}=\left\{\varphi_{t}^{\gamma}\right\}$ on $X^{\gamma}$. Then we have the following

Lemma 3. For all positive continuous $\gamma, \Phi^{\gamma}$ are weakly equivalent to each other.

Indeed $\pi(x, u)=\left(x, u \frac{r^{\prime}(x)}{r(x)}\right), 0 \leqq u<r(x)$, gives a weak equivalence between $\Phi^{\gamma}$ and $\Phi^{\gamma \prime}$.

Lemma 4. Assume $r_{0}=\inf _{x \in X_{*}} \gamma(x)>0$. For any non-trivial $\bar{\mu} \in \varepsilon\left(\Phi^{\gamma}\right)$ there exists a (unique, non-trivial) $\mu \in \varepsilon(T)$ such that

$$
E_{\bar{\mu}}(f)=\frac{1}{E_{\mu}(\gamma)} E_{\mu}\left(\int_{0}^{\gamma(x)} f(x, u) d u\right)
$$

for all continuous function $f$ on $X^{\gamma}$.

Proof. Although this lemma seems to be well known, we give here a proof of it for the completeness. The proof is analogous to the one of Proposition 1 in Section 2. Let $z=(a, 0)$ be a generic point of $\bar{\mu}$ i.e.

$$
\lim _{n, m \rightarrow \infty} \frac{1}{n+m} \int_{-m}^{n} f\left(\varphi_{t}^{\gamma} z\right) d t=E_{\tilde{\mu}}(f)
$$

for all continuous $f$. Especially we have

$$
\begin{aligned}
E_{\bar{\mu}}(f) & =\lim _{n \rightarrow \infty} \frac{1}{\sum_{n=-n}^{n} \gamma\left(T^{k} a\right)} \int_{k=-1}^{\sum_{k=0}^{n} \gamma\left(T^{k} a\right)} f\left(\sum_{k}^{\gamma} \gamma(a, 0)\right) d t \\
& =\lim _{n \rightarrow \infty} \frac{1}{\sum_{k=-n}^{n} \gamma\left(T^{k} a\right)} \sum_{k=-n}^{n} \int_{0}^{\gamma\left(T^{k} a\right)} f\left(T^{k} a, t\right) d t .
\end{aligned}
$$

Let $\mu$ be an accumulating point of probability measures $\left\{\frac{1}{2 n} \sum_{k=-n}^{n} \delta_{T^{k}} ; n>1\right\}$ on $X$ where $\delta_{x}$ denotes the point measure at $x$. Take an increasing sequence of continuous functions $g_{i}$ on $X_{*}^{\gamma}$ with compact supports such that $\lim _{i \rightarrow \infty} g_{i}=1_{X_{*}^{\gamma}}$ : Put 


$$
G_{i}(x)= \begin{cases}\int_{0}^{\gamma(x)} g_{i}(x, t) d t & x \in X_{*}, \\ 0 & x=1 .\end{cases}
$$

Since $G_{i}$ is continuous, we have

$$
\begin{aligned}
& \lim _{n^{\prime} \rightarrow \infty} \frac{1}{2 n^{\prime}} \sum_{k=-n^{\prime}}^{n^{\prime}} \int_{0}^{\gamma\left(T^{k} a\right)} g_{i}\left(T^{k} a, t\right) d t \\
= & \lim _{n^{\prime} \rightarrow \infty} \frac{1}{2 n^{\prime}{ }_{k}} \sum_{=-n^{\prime}}^{n^{\prime}} G_{i}\left(T^{k} a\right)=E_{\mu}\left(G_{i}\right)=E_{\mu}\left(\int_{0}^{\gamma(x)} g_{i}(x, t) d t\right)
\end{aligned}
$$

for some sub-sequence $\left\{n^{\prime}\right\}$, which implies together with (4) that

$$
E_{\bar{\mu}}\left(g_{i}\right)=\lim _{n^{\prime} \rightarrow \infty} \frac{1}{\frac{1}{2 n^{\prime}} \sum_{k=-n^{\prime}}^{n^{\prime}} \gamma\left(T^{k} a\right)} E_{\mu}\left(G_{i}\right) .
$$

We put

$$
c=\lim _{n^{\prime} \rightarrow \infty} \frac{1}{2 n^{\prime}} \sum_{k=-n^{\prime}}^{n^{\prime}} \gamma\left(T^{k} a\right)
$$

then

$$
1=\lim _{i \rightarrow \infty} E_{\bar{\mu}}\left(g_{i}\right)=\frac{1}{c} E_{\mu}\left(\gamma \cdot 1_{X *}\right)
$$

On the other hand, for a closed set $I_{m}^{\gamma}=\left\{(x, t) ; x \in \hat{I}_{m} \cap X_{*}, 0 \leqq t \leqq r(x)\right\} \cup\{\Delta\}$ we have

$$
\begin{aligned}
E_{\bar{\mu}}\left(1_{I_{m}^{\gamma}}^{\gamma}\right) \geqq \varlimsup_{n^{\prime} \rightarrow \infty} \frac{1}{\sum_{k=-n^{\prime}}^{n^{\prime}} \gamma\left(T^{k} a\right)} \sum_{k=-n^{\prime}}^{n^{\prime}} \int_{0}^{\gamma\left(T^{k} a\right)} 1_{I_{m}^{\gamma}}^{\gamma}\left(T^{k} a, t\right) d t \\
\geqq \frac{\gamma_{0}}{c} \lim _{n^{\prime} \rightarrow \infty} \frac{1}{2 n^{\prime}} \sum_{k=-n^{\prime}}^{n^{\prime}} 1_{\hat{I}_{m}}\left(T^{k} a\right)=\frac{\gamma_{0}}{c} \mu\left(\hat{I}_{m}\right) .
\end{aligned}
$$

Hence we have

$$
\mu(\{1\})=\lim _{m \rightarrow \infty} \mu\left(\hat{I}_{m}\right) \leqq \frac{c}{r_{0}} \lim _{m \rightarrow \infty} \bar{\mu}\left(I_{m}^{\gamma}\right)=\frac{c}{r_{0}} \bar{\mu}(\{\Delta\})=0
$$

and so $E_{\mu}(\gamma)=E_{\mu}\left(\gamma \cdot 1_{X *}\right)=c$. Therefore

$$
\begin{aligned}
E_{\bar{\mu}}(f) & =\lim _{i \rightarrow \infty} E_{\bar{\mu}}\left(f \cdot g_{i}\right)=\lim _{i \rightarrow \infty} \frac{1}{E_{\mu}(\gamma)} E_{\mu}\left(\int_{0}^{\gamma(x)} f \cdot g_{i}(x, t) d t\right) \\
& =\frac{1}{E_{\mu}(\gamma)} E_{\mu}\left(\int_{0}^{\gamma(x)} f(x, t) d t\right) .
\end{aligned}
$$


The ergodicity of $\mu$ is easily proved.

Corollary 3. If $E_{\mu}(\gamma)=\infty$ for all non-trivial $\mu \in \varepsilon(T)$, then $\delta_{\Delta}$ is the only $\Phi^{\gamma}$-invariant Borel probability measure.

Step 4. Now we will choose $r$ and $\gamma^{\prime}$ so that they prove the theorem. Take $r^{\prime}(x)=1$ on $X_{*}$ and

$$
r(x)= \begin{cases}n \cdot 4 \cdot 3^{n}, & \text { for } x \in \hat{I}_{n} \backslash \hat{I}_{n+1}, n \geqq 1, \\ 1, & \text { for } x \in X_{*} \backslash \hat{I}_{1} .\end{cases}
$$

Then using Lemma 2 we have $h\left(\Phi^{\gamma^{\prime}}\right)=h(T)>0$. On the other hand (3) implies $E_{\mu}(\gamma) \geqq n$ for all $n$ and so $E_{\mu}(\gamma)=\infty$ for all $\mu \in \varepsilon(T), \mu \neq \delta_{1}$. Therefore by Corollary 3 we see that the only $\Phi^{r}$-invariant probability measure is $\delta_{\Delta}$ and so by Lemma 2 in Section $3 h\left(\Phi^{\gamma}\right)=0$.

Remark. Similarly we can construct a pair of weakly equivalent flows one of which has finite entropy and the other has infinite entropy. However it is still unknown whether there exist such examples in the differentiable case.

Acknowledgement. I would like to thank professor $\mathrm{H}$. Totoki for helpful suggestions, especially he gave me the short proof of Lemma 1.

\section{References}

[1] Adler, R. L., Konheim, A. G. and McAndrew, M. H., Topological entropy, Trans. Amer. Math. Soc. 114 (1965), 309-319.

[2] Beboutoff M. et Stepanoff W., Sur la measure invariants dans les systems dynamiques qui ne different qui par le temps, Mat. Sb. 7 (49), (1940), 143-164.

[3] Ito, Sh., On the topological entropy of a dynamical system, Ptoc. Japan Acad., 45 (1969), 838-841.

[4] Rohlin, V. A., Entropy of metric automorphism, Dokl. Akad. Nauk USSR, 124, (1959), 980-983, (in Russian).

[5] Totoki, H., Time changes of flows, Mem. Fac. Sci. Kyushu Univ. Ser. A, 20 (1966), $27-55$.

[6] Walters, P., A variational principle for the pressure of continuous transformations, Amer. J. Math 97, N/. 4 (1975), 937-971. 